\title{
Circuit Analysis Courseware Design Based on LabView
}

\author{
Kunliang Xu1,YanLin Tao1 \\ ${ }^{1}$ Department of Computer Science and Engineering, Qujing Normal University, QuJing, 655011, \\ China
}

Keywords: LabVIEW, Circuit Analysis, Courseware

\begin{abstract}
As LabView offers a perceptually direct interface and good interactivity as well as a strong and flexible mathematical analysis function, which is helpful in circuit analysis where the changes of various physical quantities are hard to observe directly, this research has made with LabView several courseware presentations covering teaching contents such as linear resistance circuits, dynamic circuits and sinusoidal current circuits so as to help teachers explain these knowledge and students observe the changes of the physical quantities directly in learning and understand circuits better.
\end{abstract}

\section{Introduction}

A circuit has an important status in electronic information technology, the main content of which is to analyze various electromagnetic phenomena and processes in circuits and explain the basic law of circuits and the circuit analysis methods. To learn this course that involves complex components and circuits, the key is to understand and study the connotations of the law of various circuit theorems, and observe circuit phenomena from all kinds of circuit experiments, and analyze them using theories. Changes of various physical quantities in circuits, however, are invisible and imperceptible, not easy to observe directly, which has caused certain difficulties for understanding[1].

Most teachers now use multimedia courseware in teaching. Although there are certain effects[2], but the parameters in the multimedia courseware cannot directly be set up to analyze the circuits, causing poor interactivity. This paper, based on the features of the LabView, developed the courseware for basic circuit analysis. Using the courseware for teaching, the effect is better[3-5].

\section{Introduction of LabVIEW development tool}

LabVIEW (Laboratory Virtual Instrument Engineering Workbench) is a powerful graphical virtual instrument development environment, widely accepted by industries and research institutions in automobiles, semiconductors, aviation and aerospace, transportation, telecommunication and biological medicine, etc. As a typical graphical programming language (G language), its development environment combines the high performance and flexibility of graphical programming formulas with the high-tech and configuration targeted at testing, measurement and automated control. It provides the necessary development tools for various applications including data collection, instrument control, measurement, analysis and data display, etc [6].

LabVIEW programming environment boasts of many advantages: the structured programming does not require much code-writing; it uses familiar terms, icons and concepts; it provides numerous clear icons, expandable function libraries and subroutine libraries. Using it, program design and function can be quite easy; principle research, design, testing and equipment system implementation can be far more efficient. Therefore, with LabVIEW, virtual platform designing can be much less work-load and lower costs [7].

\section{Main interface design of the courseware}

Main interface is composed of linear resistance circuit analysis, dynamic analysis, and circuit characteristic analysis, where linear resistance circuit analysis includes three modules-branch current method, loop current method and node voltage method; the temporal logic circuit includes 
two modules-first order dynamic circuit analysis, and second-order dynamic circuit analysis; circuit characteristic analysis includes three modules-the sinusoidal current circuit vector characteristics, frequency characteristics, and resonant circuit characteristic analysis. In total there are eight modules. The details are shown in Fig.1 below.

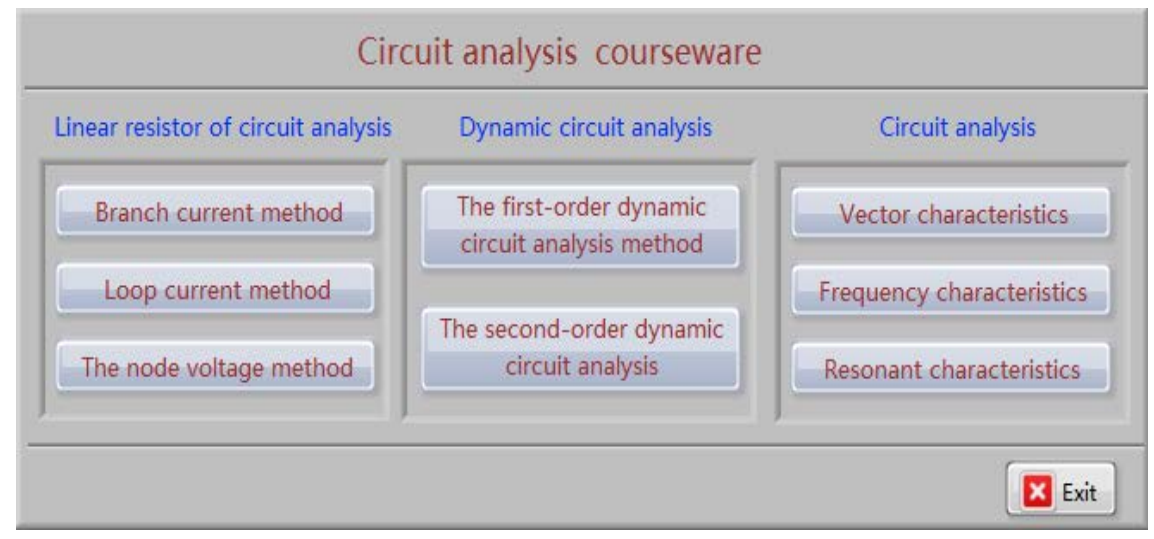

Fig.1 Front panel of the main interface of the courseware for circuit analysis

In the main interface in the diagram, the conditional structure is used to control program exit; the event structure is used to select among experimental projects; after choosing a certain experimental project, the sub-form can be started by calling the sub VI. This process involves functions of the creation of path, the attribute node, opening VI reference, calling nodes, closing reference etc. The specific program chart is shown in Fig.2.

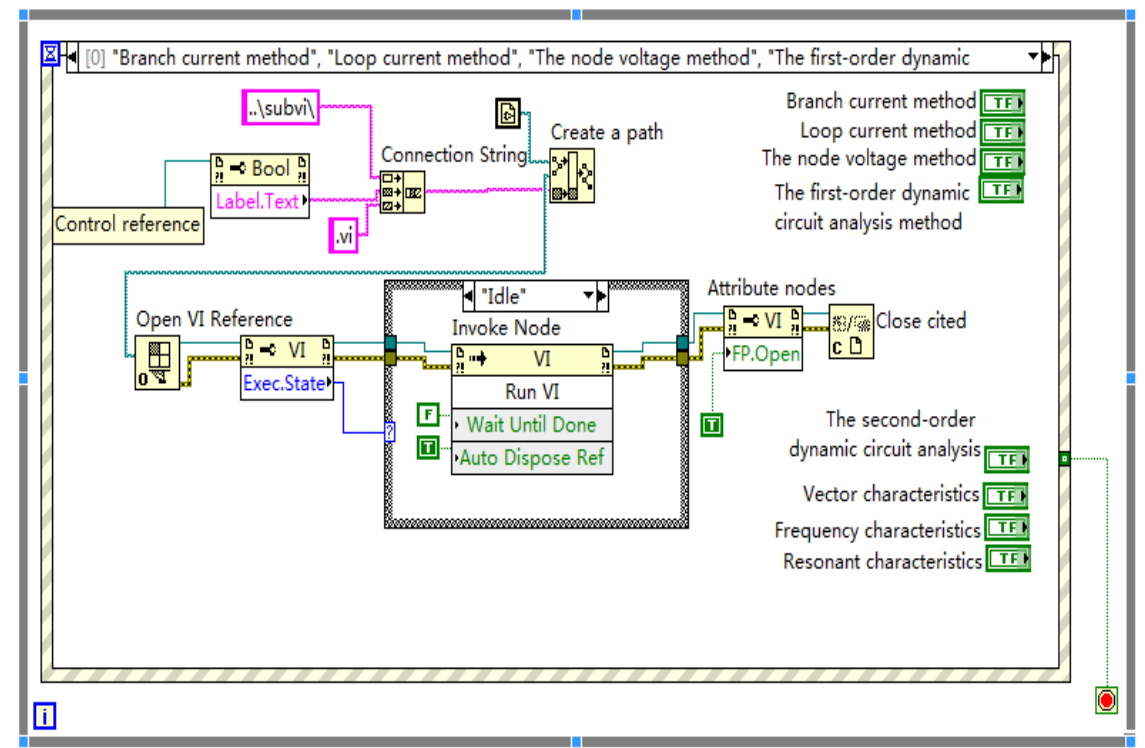

Fig.2 The program chart of the main interface of the courseware for circuit analysis

\section{Sample design for circuit analysis}

The essay focuses on the detailed introduction of the design process of branch current method and loop current method in linear resistance circuit analysis.

\section{Branch current method instance}

Where resistance and supply voltage are known, according to the circuit diagram shown in Fig.3 below. 


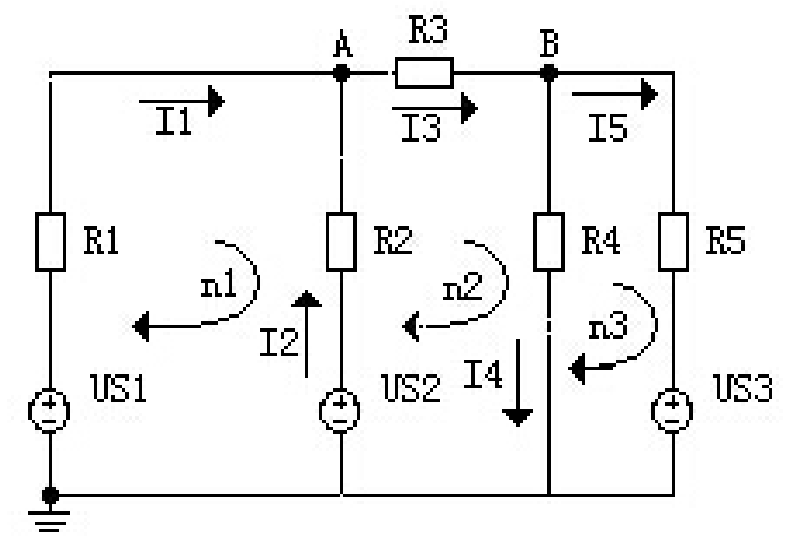

Fig.3 Branch current method of circuit analysis

using branch current method, the KVL equations of A and B nodes are as follows:

$$
\left\{\begin{array}{l}
-I_{1}-I_{2}-I_{3}=0 \\
-I_{3}-I_{4}+I_{5}=0
\end{array}\right.
$$

Formulate KVL equations for n1, n2 and n3, then

$$
\left\{\begin{array}{c}
R_{1} I_{1}-R_{2} I_{2}=U_{s 1}-U_{s 2} \\
R_{2} I_{2}+R_{3} I_{3}+R_{4} I_{4}=U_{s 2} \\
-R_{4} I_{4}+R_{5} I_{5}=-U_{a 3}
\end{array}\right.
$$

Make them into the matrix equation set:

$\left[\begin{array}{ccccc}-1 & -1 & 1 & 0 & 0 \\ 0 & 0 & -1 & 1 & 1 \\ R_{1} & -R_{2} & 0 & 0 & 0 \\ 0 & R_{2} & R_{3} & R_{4} & 0 \\ 0 & 0 & 0 & -R_{4} & R_{5}\end{array}\right]\left[\begin{array}{l}I_{1} \\ I_{2} \\ I_{3} \\ I_{4} \\ I_{5}\end{array}\right]=\left[\begin{array}{l}0 \\ 0 \\ U_{s 1}-U_{s 2} \\ U_{s 2} \\ -U_{s 3}\end{array}\right]$

Based on the above matrix equation, the front panel is designed as shown in Fig. 4 below:

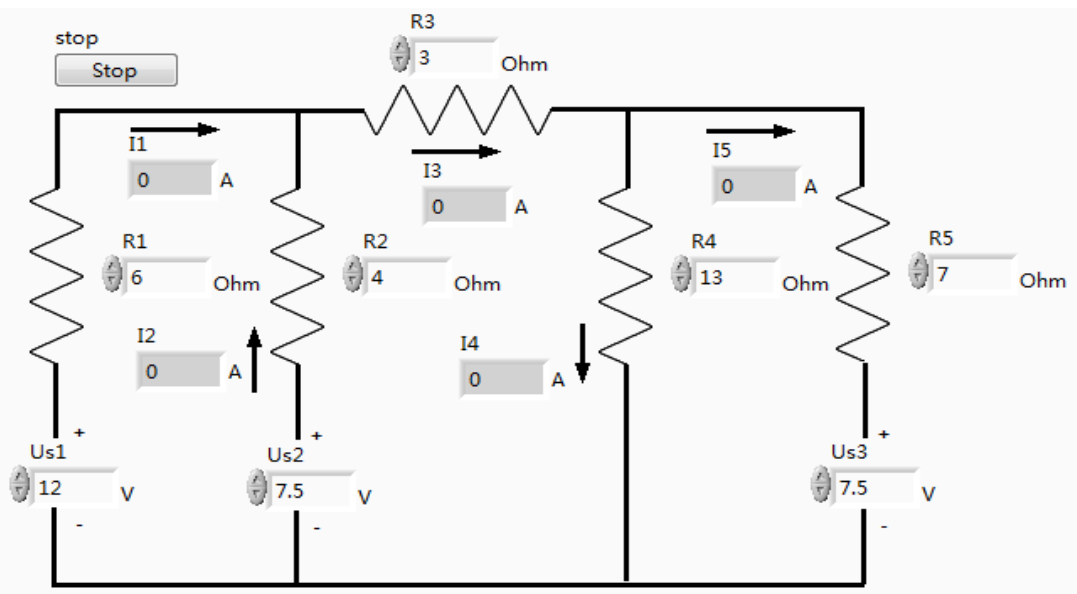

Fig.4 The front panel of "branch current method. vi"

Using MathScript node, the coefficient matrices and the known vectors of the linear equation set are obtained. The solution vector is then obtained with the linear equation set Linear Equation. Vi. The element values of the vector are the current values of $I_{1}-I_{5}$. The completed program chart is shown in Fig.5 below[8]. 


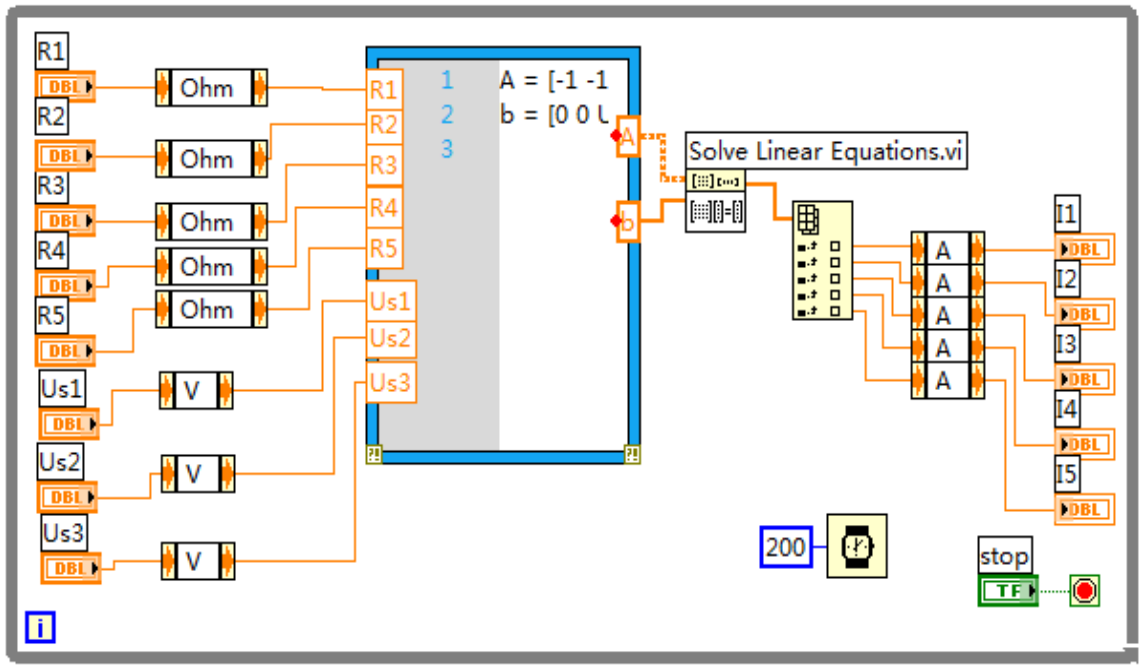

Fig.5 The program chart of "branch current method. Vi"

\section{Loop current method instance}

Circuit shown in figure 3, for example,using loop current method, the dotted line in the figure shown in three circuits, lists the circuit voltage equation are as follows:

$$
\left\{\begin{array}{c}
\left(R_{1}+R_{2}\right) I_{n 1}-R_{2} I_{n 2}=U_{s 1}-U_{s 2} \\
-R_{2} I_{n 1}+\left(R_{2}+R_{3}+R_{4}\right) I_{n 2}-R_{4} I_{n 3}=U_{s 2} \\
-R_{4} I_{n 2}+\left(R_{4}+R_{5}\right) I_{n 3}=-U_{s 3}
\end{array}\right.
$$

$I_{n 1} 、 I_{n 2} 、 I_{n 3}$ for each loop current. Rewrite the forms for the linear equations are:

$\left[\begin{array}{ccc}R_{1}+R_{2} & -R_{2} & 0 \\ -R_{2} & R_{2}+R_{3}+R_{4} & -R_{4} \\ 0 & -R_{4} & R_{4}+R_{5}\end{array}\right]\left[\begin{array}{l}I_{n 1} \\ I_{n 2} \\ I_{n 3}\end{array}\right]=\left[\begin{array}{c}U_{s 1}-U_{s 2} \\ U_{s 2} \\ -U_{s 3}\end{array}\right]$

Based on the above matrix equation, the front panel is designed as shown in Fig. 6 below:

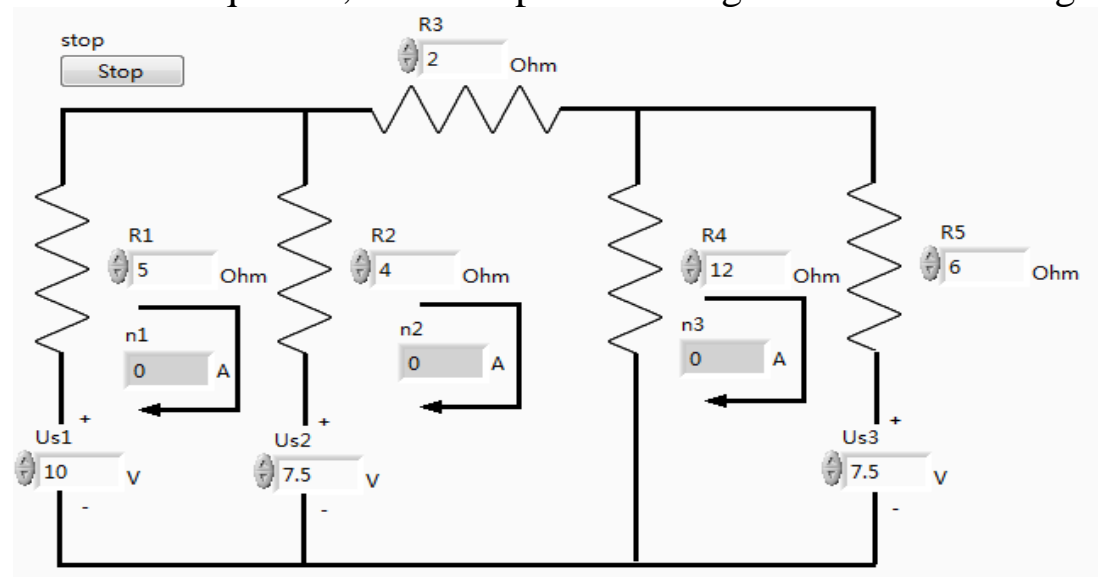

Fig.6 The front panel of "loop current method. vi"

With reference to the case method,still using MathScript node, the coefficient matrices and the known vectors of the linear equation set are obtained. The solution vector is then obtained with the Solve Linear Equations.vi. The element values of the vector are the current values of $I_{n 1}-I_{\mathrm{n} 3}$. The completed program chart is shown in Fig.7 below. 


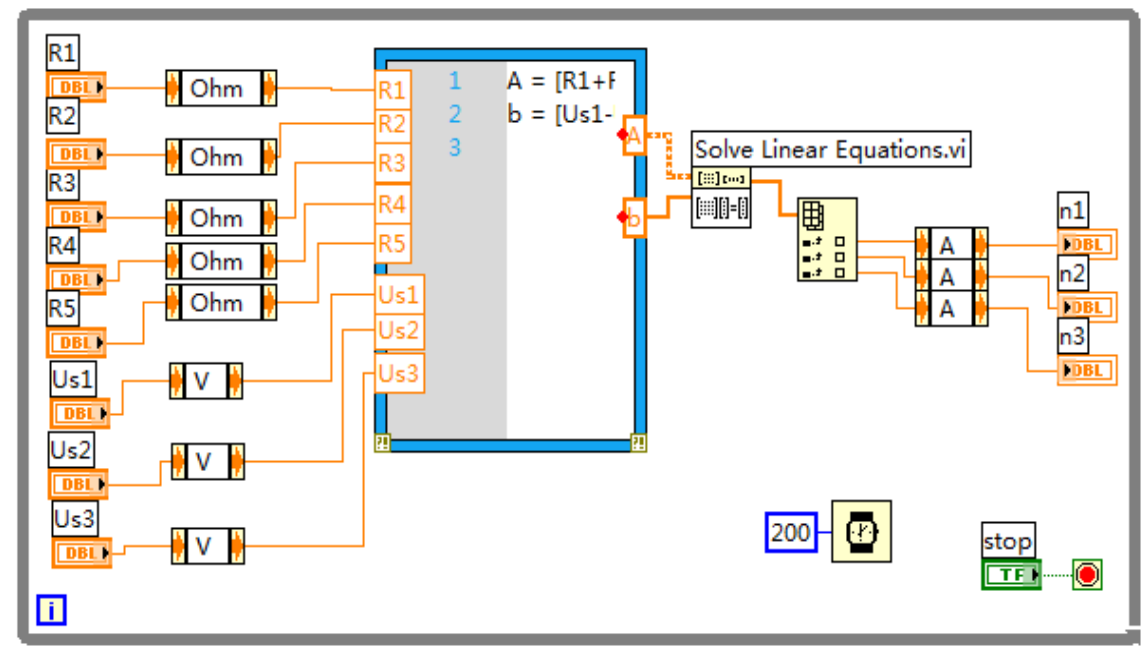

Fig.7 The program chart of "loop current method. Vi"

\section{Conclusion}

Teaching with this courseware can help teachers reduce difficulty in explanation and enhance perceptibility, and allow students to self-study with the courseware and make virtual experiments. Students can have an overall understanding and command of the methods of circuit analysis and design in electric technology through the adjustment of parameters, repetition of experiments, comparisons and analyses, so that they can connect different knowledge learned in various courses, improve creativity, raise learning motivation, increase their initiatives and activeness in participation and lay a solid foundation for later works.

\section{References}

[1] D.J. HE. Effective Teching Discussion and Analysis on the Circuit Analysis Course .Journal of HuaiHua University, 301(15), pp.79-81. 2011.

[2] Y.H. Hou, Y.E. Wang. Teaching Design of course Circuit Analysis.Journal of Shaanxi Institute of Technology, 24(2),pp.9-13. 2014.

[3] W.T. WANG,J.C. ZHAO,Z.F. GU,Z.C. LIU. Application of LabVIEW in Teaching of Circuit Analysis.Experiment Science and Technology, 12(3),pp.49-52. 2014.

[4] Y.P. DAI. Application analysis on Experiment Teaching of Educational Technology Using LabVIEW platform.Modern Educational Technology, 22(5),pp.113-117. 2012.

[5] G.X. Cai. Application of LabVIEW in Multimedia Courseware Design .Journal of Southwest China Normal University(Natural Science Edition), 35(4),pp.155-158. 2010.

[6] Information on http://china.ni.com/

[7] T.Zhang,G.S. Chen, Z.L. Wang:Proficient in Labview programming, Beijing: Electronic industry press, 2008.

[8] J.Q. Zhang, D.Y. Chen, Y. Lu. Applications of LabVIEW in dynamic circuit analysis experiment .Journal of Xi'An University of posts and Telecommunications, 16 (s1),pp. 87-89. 2011. 